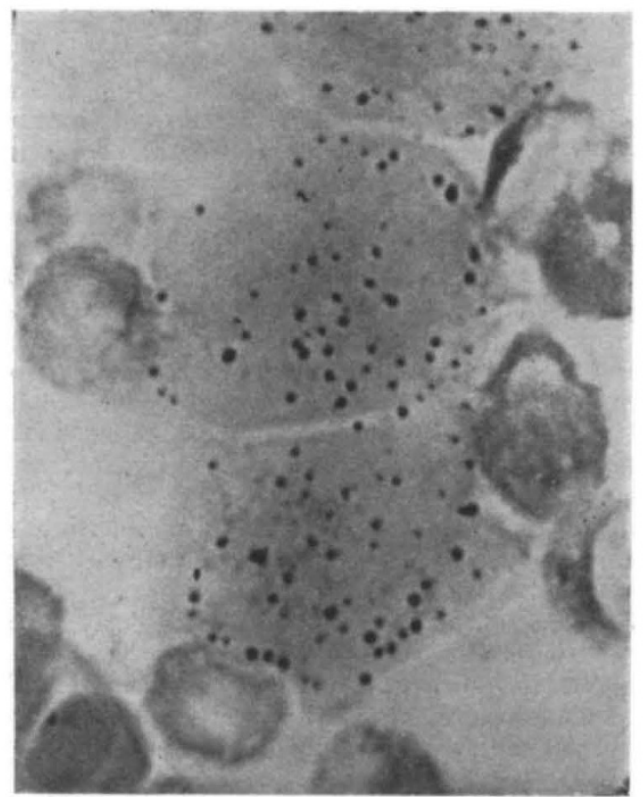

Fig. 2. Scattered granular positivity in leukæmic monocytes.

staining with sudan black $B$ is not, however, disturbed, since appearances after acetone fixation are similar to those after formalin fixation.

Patterns of succinic dehydrogenase distribution in lymphocytes and monocytes are shown in Figs. 1 and 2. Granulocyte precursors up to the stage of myolocytes and metamyelocytes also showed sites of enzymatic activity, but polymorphs were predominantly negative in the spocimens we have studied hitherto.

Department of Medicine,

Dennis Quaglino

F. G. J. HAYHOE

University of Cambridge.

1 Tsou, K.-C., Cheng, C.-S., Nachlas, M. M., and Seligman, A. M. J. Amer. Chem. Soc., 78, 6139 (1956).

${ }^{2}$ Nachlas, M. M., Tsou, K.-C., de Souza, E., Cheng, C.-S., and Seligman, A. M., J. Histochem. Cytochem., 5, 420 (1957).

${ }_{3}$ Le Souza, E., and Kothare, S. N., J. Histochem. Cytochem., 7, 77 (1959).

4Willighagen, R. G. J., and Planteydt, If. T., Nature, 183, 263 (1959)

'Novikoff, A. B., Shin, W.-Y., and Drucker, J., J. Histochem. Cytochem., 8, 37 (1960).

\section{Absorbance of Feulgen-stained Somatic Cell Nuclei}

ANALYSIS of published data on cytophotometric measurements of Feulgen-stained somatic cell nuclei from rat liver and thyroid gland ${ }^{1}$, untreated and croton oil treated mouse ear epithelial nuclei ${ }^{2}$ and mouse neuron and liver nuclei ${ }^{3}$ has shown that a straight line is obtained when the absorbance, $E$, is plotted against the reciprocal of the squared nuclear diameter. Consequently $a$ and $b$ are constants in the expression :

$$
E=a+b D^{-2}
$$

The ways in which the total nuclear deoxyribonuclcic acid-content have been calculated ${ }^{\text {s-8 }}$ demonstrate that the nuclei have been imagined as being homogeneously absorbing objects, that is, objects in which the absorbing particles are randomly distributed, non-interacting and retaining their shape and number. But according to the equation the mass (=deoxyribonucleic acid-content) must remain constant, no synthesis can therefore take place, and this is contradictory to experimental facts. We can therefore conclude that in spite of the finding that the equation describes the data well, it does not mean that the nuclei are homogeneously absorbing objects. A non-random distribution of the Feulgen-stain was observed by DeSmul and LeComte, and reconstructions of serially cut human and rodent somatic nuclei in this laboratory ${ }^{10}$ have shown that the Feulgenstained material and the nucleoli are distributed in a peripheral zone. We can therefore conclude that the absorbing material, that is, deoxyribonucleic acid, is not randomly distributed and consequently that published data on the total nuclear deoxyribonucleic acid contont, calculated from cytophotometric measurements of fixed tissue assuming a random distribution of the absorbing particles, are erroneous. 'The linear relationship between the nuclear absorbance and the inverse nuclear surface area may simply mean that both the absorbance and the geometrical dimensions are dependent variables of a common variable as, for example, the growth process, thus :

$$
E=f_{1}(X) \text { and } D^{2}=f_{2}(X)
$$

A practical consequence of this is, as is also ovident from the equation, that a simple measurement of the nuclear geometrical dimensions will yield the same information as that obtained by the far more complicated and costly photometric method.

The numerical value of the constant $b$, which may well have different absolute values for different tissues, is thus not a direct measure of mass, but is a measure of the rate at which the absorbance decreases for unit surface area increase over a given mass range. For nuclei retaining their ploidy the growth process results in a doubling of the chromatin mass (deoxyribonucleic acid) and an increase in size between two consecutive divisions. As the relative mass of nuclei of different ploidy has the value of $1: 2: 4 \ldots$, it follows that for a stationary population of nuclei of different ploidy the relative numerical values of the constant $b$ will show this ratio. If transitions from one nuclear class to another occur the relative value of $b$ will depend upon the intensity of transition. Deviations in the relative values of $b$ from $1: 2: 4 \ldots$ can accordingly be interpreted as a measure of the degree of cell differentiation. We would therefore expect, as also found by Moore ${ }^{11}$, that the degree of deviation from the ratio of $1: 2: 4$ varies from individual to individual as well as with age.

\section{SIMON IVERSEN}

Cancer Research Department, Royal Beatson Memorial Hospital,
Glasgow.

$$
\text { Fob. } 29 .
$$

${ }^{1}$ Alfert, M., Bern, H. A., and Kahn, R. H., Acta Anat., 23/24, 185 (1955).

${ }^{2}$ Pasteels, J., and Bullough, W. S., Arch. Biol. (Paris), 64, 271 (1953). ${ }^{3}$ Swift, H., Physiol. Zool., 23, 189 (1950).

${ }^{4}$ Swift, H,, Int, Rev, Cytol., 2, 1 (1953).

"Swift, H., and Rasch, E., "Physical Techniques in Blol. Res.", 3, 353 (1956).

- Kurnick, N. B., Int. Rev. Cytol., 4, 221 (1955).

"Vendrely, R., and Vendrely, C., Int. Rev. Cytol., 5, 171 (1956).

${ }^{8}$ Lcuchtenberger, C., "General Cytochemical Methods" (1958).

- DeSmul, A., and LeComte, C., Arch. Biol. (Liège), 64, 250 (1953).

${ }^{10}$ Yversen, S., Acta Anat. (In the press).

"I Noore, B. C., Chromosoma, 4, 565 (1952). 